\title{
Gestion des dysplasies osseuses florides symptomatiques : série de cas et revue de littérature
}

\author{
M. Bouhoute (Rabat), K. El Harti (Rabat), W. El Wady (Rabat)
}

Introduction : Les dysplasies osseuses sont définis (WHO histological classification of odontogenic tumours 2005) comme des processus idiopathiques localisés au niveau de la région périapicale des dents, caractérisées par le remplacement de l'os normal par un tissu fibreux avec un os métaplasique. Ces conditions regroupent différentes formes cliniques : dysplasie osseuse périapicale, localisée au niveau des apex des dents antérieures, dysplasie osseuse focale, localisée aux dents postérieures, dysplasie osseuse floride (DOF) touchant 2 à 4 quadrants et cemontome gigantoforme familial, présent chez le jeune avec une symptomatologie très marquée. La découverte de ces lésions est souvent fortuite au niveau de radiographie panoramique avec des images radioclaires, mixtes ou radio-opaques, de différentes tailles. Cependant, une symptomatologie infectieuse peut être associée à ces lésions. Observations : Les quatre cas cliniques reportés représentent différentes situations pouvant révéler la dysplasie osseuse floride. Le premier cas clinique d'une patiente âgée de $56 \mathrm{ans}$ avec une petite dénudation osseuse de $3 \mathrm{~mm}$ au niveau du quadrant3 avec une tuméfaction endobuccale douloureuse comblant le vestibule. La lésion est fréquemment traumatisée par une PAT mandibulaire. Le deuxième cas clinique d'une patiente de 50ans avec une tuméfaction exobuccale gauche avec une peau de recouvrement d'aspect normal, légèrement sensible à la palpation et faisant suite à l' extraction de la 36 datant de 2 mois, le 3 ème et 4ème cas cliniques sont de deux femmes de 50 et 70 ans, se présentant avec des fistules cutanées accompagnées de tuméfactions exobuccales gauches. Discussion : Pour les 4 cas cliniques, le diagnostic de dysplasie osseuse floride compliquée d'une infection est posé suite à une investigation clinique et radiologique minutieuse, leurs gestion est chirurgicale avec une bonne évolution dans le temps. La littérature objective que la symptomatologie de la DOF reste rare et lorsqu'elle existe, elle se manifeste le plus souvent par une tuméfaction endobuccale, accompagnée d'une douleur comme pour le premier cas clinique ; les recherches considèrent que l'infection est secondaire à l'exposition des masses pathologiques après résorption des corticales alvéolaires chez le sujet édenté ou après extraction de dents proches des lésions chez le denté. Le 2ème cas clinique peut porter appui à ces résultats, vu que l'infection secondaire peut être rattachée à l'extraction de la 36. La diminution de la vascularisation au niveau des sites atteints expose le patient à un risque élevé de diffusion de l'infection se manifestant par une ostéomyélite voire une cellulite si la prise en charge est retardée ou non adéquate comme objectivé pour les 3ème et 4ème cas cliniques, avec une ostéomyélite accompagnée d'une extériorisation de l'infection sous forme de fistule cutanée. Conclusion : Une attention particulière devra être apportée à la prise en charge chirurgicale qui risque d'exposer plus de tissu vasculaire et entrainer des complications. La prise en charge adoptée dans ces cas cliniques répond à celle recommandée par la littérature avec exérèse des tissus responsables de l'infection, accompagnée d'un débridement des sites d'ostéomyélite avec une abstention et surveillance pour les sites asymptomatiques. 